\title{
Optimizing age-specific vaccination
}

\author{
By Meagan C. Fitzpatrick ${ }^{1,2}$ and Alison P. Galvani ${ }^{2}$ \\ ${ }^{1}$ Center for Vaccine Development and Global Health, University of Maryland School of Medicine, Baltimore, MD, USA. ${ }^{2}$ Center for Infectious Disease Modeling and Analysis, \\ Yale School of Public Health, New Haven, CT, USA. Email: alison.galvani@yale.edu
}

\section{Vaccination strategies are not one size fits all}

Efficacious vaccines against severe acute respiratory syndrome coronavirus 2 (SARS-CoV-2) have been developed, tested, and approved for emergency use with unprecedented speed. Deployment of multiple vaccines was initiated in several countries less than 1 year after identification of the virus. Although vaccine production is being rapidly scaled up, demand will exceed supply for the next several months. Consequently, an urgent challenge is the optimization of vaccine allocation to maximize public health benefit. Bubar et al. (I) demonstrate that vaccination of older people is the optimal age-based strategy to alleviate the burden of COVID-19. Although vaccination of younger adults is projected to avert the greatest incidence, vaccinating older adults will most effectively reduce mortality. In addition, they assess targeted vaccination that was based on serological status, finding that vaccinating seronegative individuals improves efficiency especially in settings where seroprevalence is high.

Some have looked to influenza vaccination strategies for guidance, given that SARS-CoV-2 is a respiratory virus with similar modes of transmission (2). Until 2008, the U.S. Centers for Disease Control and Prevention (CDC) prioritized people aged 50 years and older for influenza vaccination because of their increased risk of mortality (3). To evaluate this long-standing policy, optimal influenza vaccine allocation was determined on the basis of a dynamic transmission model combined with optimization algorithms that facilitated the searching of wide ranges of parameter space across multiple dimensions concurrently (4). The dynamic model component simulates both the direct protection conferred to a vaccinated individual as well as the indirect protection for others that is attributable to reductions in onward transmission. This modeling indicated that allocating scarce vaccines to school-age children and young adults achieves the most effective reduction in influenza burden whether measured in terms of incidence, hospitalizations, years of life lost, or economic cost (4). These insights prompted an expansion of recommendations to prioritize school-age children (5), which interrupts transmission for all ages and bolsters indirect protection against mortality. Bubar et al. extend the framework of transmission modeling combined with vaccine optimization developed for influenza (4) to explore SARS-CoV-2 vaccination strategies and, different from the optimal influenza vaccination strategy, they conclude that vaccinating older people most effectively mitigates mortality from COVID-19.

Although it may seem intuitive that the optimal strategies against influenza and COVID-19 would be identical, vaccine optimization is not one size fits all, even for apparently similar pathogens. Quantitative changes in the epidemiological landscape can lead to qualitative shifts in optimization (see the figure). The age-specific probability of infection is a key component of this landscape. As a result of contact patterns, older people are simultaneously less exposed to and less responsible for onward transmission of both influenza virus and SARS-CoV-2 (6). An awareness of their heightened risk for severe clinical outcomes from COVID-19 has further suppressed contacts of older people. By contrast, essential workers, who are more likely to be exposed to SARS-CoV-2, are usually younger adults. These behavioral factors point to reduced public health benefit from vaccination of older people, but there are other factors that must also be considered.

Vaccine efficacy also affects optimization. Vaccines that elicit more efficacious direct protection against infection concomitantly generate stronger indirect effects (7). Highly efficacious vaccines administered to younger people can efficiently interrupt transmission chains, whereas modestly efficacious vaccines do not. With everything else being equal, vaccine efficacy as high as so far reported for SARS-CoV-2 vaccines also favors prioritization of younger people $(7,8)$. A caveat is that clinical trials for SARS-CoV-2 vaccines have measured protection against symptomatic disease, not necessarily infection. If these vaccines are less efficacious against infection and onward transmission, indirect protection would be diminished, and vaccination of older people would be consistently optimal.

However, the magnitude of two other elements counterbalances the influence of age-specific contact rates and high vaccine efficacy on optimal vaccination strategies: overall infectiousness and differences in case fatality according to age. Vaccines are less able to prevent death through indirect protection when infectiousness rises because the virus is able to infect more unvaccinated people. SARS-CoV-2 is highly contagious: Its reproductive number, $R_{0}$ (the average number of secondary infections arising from a single case when everyone is assumed to be susceptible), is typically double that of influenza virus $(7,9)$. Furthermore, age-based differences in case fatality are much larger for COVID-19 than for influenza. 
For example, hospitalized individuals aged 71 to 80 years have 46.2 times the risk of mortality compared with those aged 18 to 30 years, whereas this parameter is 9.3 times for seasonal influenza (10). Indirect effects from vaccinating younger people are unlikely to compensate for the amplified direct risk posed to older people by COVID-19. Beyond overall efficacy, vaccine characteristics can affect optimal vaccination strategies. Influenza vaccines have variable age-specific efficacy, with reduced protection for older people compared with younger individuals (7). Interim data from an ongoing phase III clinical trial of a SARS-CoV-2 vaccine do not indicate a comparable discrepancy because the vaccine appears to have similar efficacy across age groups (8).

An additional consideration relates to the age-specific effectiveness of nonpharmaceutical interventions (NPIs). Some measures, including masks and movement restrictions, reduce transmission risk regardless of symptom status. Others, such as temperature checks, are symptom-based and therefore miss asymptomatic infections. When the severity of symptoms correlates with age, such as for COVID-19 and influenza, there is a parallel correlation in the effectiveness of symptom-based interventions. Given that young people are more likely to be asymptomatic (11), infections in this group are less likely to be contained by symptom-based control but are still able to be prevented by vaccination. Under these conditions, the delayed vaccination of young people would increase the ratio of asymptomatic to symptomatic infections. Such silent transmission has driven the widespread dissemination of SARS-CoV-2 (12). Although these considerations are unlikely to greatly alter prioritization for COVID-19 vaccines, public health officials should be mindful of these changing dynamics during the period when many older individuals are vaccinated and many younger people are not. It is imperative to maintain NPIs until high vaccination coverage is attained in all age classes.

The complexity of these epidemiological, clinical, behavioral, and vaccine-related relationships underscores the importance of pathogen-specific transmission modeling to inform vaccination strategies. Such models have guided vaccination policies for myriad diseases, including those caused by rubella virus, human papillomavirus, and varicella-zoster virus, which exhibit age-specific patterns in both transmission and severity. This framework can also be applied to decisions about allocation that are not based on age but in which there are analogous dichotomies between mortality and transmission potential. At the same time, it is imperative to remain cognizant that model predictions are only as accurate as the data on which they are based. A particular limitation of modeling during an emerging disease outbreak is the sparsity of data (13). Current sources of imprecision for modeling the COVID-19 pandemic include evolving age-specific behavioral patterns as well as the temporal and geographical heterogeneity in adoption of NPIs. Also, a persistent pitfall for modeling is that analyses that galvanize action to avert crisis necessarily make themselves obsolete (13).

Bubar et al. elegantly synthesize the available data for COVID-19 within their model, concluding that the age-based strategy that saves the most lives is vaccination of older individuals. Both SARS-CoV-2 and influenza virus vaccination can be optimized within the overarching paradigm that balances the indirect risks of transmission against direct risks from mortality. Although vaccinating younger people prevents the most infections for both viruses, the difference is that this strategy does not also avert the most deaths for COVID-19. To vanquish a pathogen that causes such steeply divergent case fatality rates as that of SARS-CoV-2, the optimal strategy is clear: Directly vaccinate those with greatest personal risk.

\section{REFERENCES AND NOTES}

1. K. M. Bubar et al., Science 10.1126/science.abe6959 (2021).

2. K. Tingley, New York Times, 5 November 2020; www.nytimes.com/2020/11/05/magazine/who-gets-covid-vaccine.html.

3. A. E. Fiore, D. K. Shay, K. Broder, J. K. Iskander, T. M. Uyeki, G. Mootrey, J. S. Bresee, N. S. Cox; Centers for Disease Control and Prevention (CDC); Advisory Committee on Immunization Practices (ACIP), Prevention and control of influenza: Recommendations of the Advisory Committee on Immunization Practices (ACIP), 2008. MMWR Recomm. Rep. 57 (RR-7), 1-60 (2008). Medline

4. J. Medlock, A. P. Galvani, Optimizing influenza vaccine distribution. Science 325, 1705-1708 (2009). doi:10.1126/science.1175570 Medline

5. A. E. Fiore, T. M. Uyeki, K. Broder, L. Finelli, G. L. Euler, J. A. Singleton, J. K. Iskander, P. M. Wortley, D. K. Shay, J. S. Bresee, N. J. Cox; Centers for Disease Control and Prevention (CDC), Prevention and control of influenza with vaccines: Recommendations of the Advisory Committee on Immunization Practices (ACIP), 2010. MMWR Recomm. Rep. 59(RR-8), 1-62 (2010). Medline

6. E. Zagheni, F. C. Billari, P. Manfredi, A. Melegaro, J. Mossong, W. J. Edmunds, Using time-use data to parameterize models for the spread of close-contact infectious diseases. Am. J. Epidemiol. 168, 1082-1090 (2008). doi:10.1093/aje/kwn220 Medline

7. P. Sah, J. Medlock, M. C. Fitzpatrick, B. H. Singer, A. P. Galvani, Optimizing the impact of low-efficacy influenza vaccines. Proc. Natl. Acad. Sci. U.S.A. 115, 51515156 (2018). doi:10.1073/pnas.1802479115 Medline

8. F. P. Polacket al., N. Engl. J. Med. 10.1056/NEJMoa2034577 (2020)

9. J. Hilton, M. J. Keeling, Estimation of country-level basic reproductive ratios for novel Coronavirus (SARS-CoV-2/COVID-19) using synthetic contact matrices. PLOS Comput. Biol. 16, e1008031 (2020). doi:10.1371/journal.pcbi.1008031 Medline

10. L. Pirothet al., Lancet Respir. Med. 10.1016/S2213-2600(20)30527-0 (2020).

11. N. G. Davies et al., Nat. Med. 10.1038/s41591-020-0962-9 (2020).

12. S. M. Moghadas et al., Proc. Natl. Acad. Sci. U.S.A. 10.1073/pnas.2008373117 (2020).

13. M. C. Fitzpatrick, C. T. Bauch, J. P. Townsend, A. P. Galvani, Modelling microbial infection to address global health challenges. Nat. Microbiol. 4, 1612-1619 (2019). doi:10.1038/s41564-019-0565-8 Medline

Published online 21 January 2021

10.1126/science.abg2334 


\section{Key determinants of age-based vaccination}

Epidemiological factors drive age-based vaccine optimization for influenza and COVID-19 with varying influence according to the mechanism and magnitude of the factor. When there are stark differences in mortality risk with age, or when the reproductive number $R_{0}$ is high, older people are more likely to be prioritized for vaccination. By contrast, high vaccine efficacy, the amount of social contact among younger people, and widespread adherence to nonpharmaceutical interventions can shift prioritization toward younger ages.

Influenza COVID-19

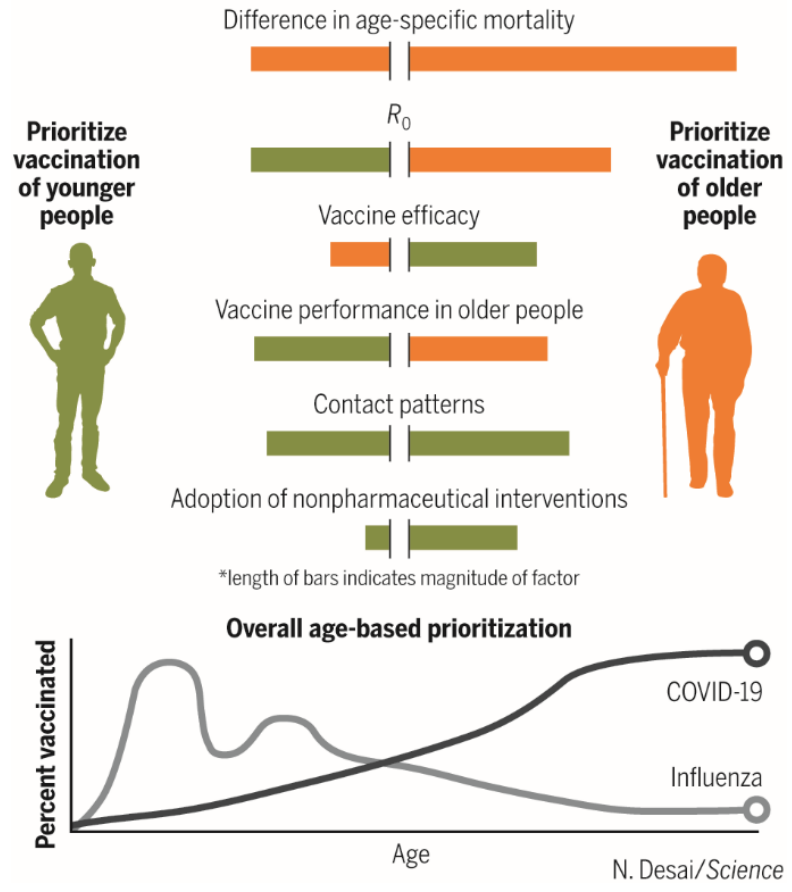

